FORMATION Formation emploi

Revue française de sciences sociales

$105 \mid$ janvier-mars 2009

Pêle-mêle

\title{
Discriminations dans l'accès au stage : du ressenti des élèves à l'intervention des enseignants
}

Diskriminierungen beim Zugang zu Berufspraktika: Vom Eindruck der Schüler bis zum Einschreiten der Lehrer

Discrimination in internship acceptance: from student perceptions to teacher support

Discriminaciones en el acceso a las pasantías : de la vivencia de los estudiantes a la intervención de los docentes

\section{Nicolas Farvaque}

\section{(2) OpenEdition} Journals

Édition électronique

URL : http://journals.openedition.org/formationemploi/1803

DOI : 10.4000/formationemploi.1803

ISSN : 2107-0946

Éditeur

La Documentation française

Édition imprimée

Date de publication : 1 mars 2009

Pagination : 21-36

ISSN : 0759-6340

Référence électronique

Nicolas Farvaque, « Discriminations dans l'accès au stage : du ressenti des élèves à l'intervention des enseignants », Formation emploi [En ligne], 105 | janvier-mars 2009, mis en ligne le 01 mars 2011, consulté le 10 décembre 2020. URL : http://journals.openedition.org/formationemploi/1803; DOI : https://doi.org/10.4000/formationemploi.1803

(c) Tous droits réservés 


\title{
Formation
}

\section{Discriminations dans l'accès}

\section{au stage : du ressenti des élèves à l'intervention des enseignants}

\author{
par Nicolas Farvaque*
}

L'article souligne les difficultés propres à ces premières confrontations avec le marché du travail que représentent les stages pour les lycéens en lycée professionnel, et les modalités de réponse des enseignants. Les facteurs liés à l'origine et au milieu social se cumulent pour expliquer le sentiment de difficulté et le recours à l'aide de l'établissement.

Les stages en entreprise ont une fonction reconnue de formation professionnelle et de signal sur le marché $\mathrm{du}$ travail ${ }^{1}$. Les entreprises peuvent recourir à ces stages de diverses façons, des stages pouvant être formateurs ou se substituer à des emplois plus classiques (Domingo, 2002). Dans ce dernier cas, certaines dérives dans l'utilisation des stages en entreprise ont récemment été dénoncées. Il s'agissait principalement des stages effectués par les étudiants après le baccalauréat.

Les stages interviennent toutefois bien plus tôt dans les parcours scolaires. Nous nous intéresserons ainsi aux stages réalisés dans l'enseignement secondaire,

\footnotetext{
${ }^{1}$ L'article est issu d'une recherche réalisée par l'ORSEU et financée dans le cadre du projet Transfert ( Transfert de pratiques pour un Accès Non Discriminatoire au Stage et à la Formation professionnelle - Éducation en Réseau pour la Tolérance ») du programme européen Equal (Fonds social européen), coordonné par la Fédération nationale Léo Lagrange et l'Union nationale des syndicats autonomes (UNSA).
}

essentiellement dans les filières professionnelles mises en place au niveau des lycées, tout en considérant en contrepoint le cas de certaines formations post-bac organisées au niveau des lycées (BTS brevet de technicien supérieur). Pour ces lycéens et étudiants, l'accès au stage est une question centrale, la qualité du stage intervenant grandement sur la

* Nicolas Farvaque est directeur du pôle « Territoires et Emploi - Recherche et études » à l'ORSEU (Office européen de conseil, recherche et formation en relations sociales), Lille. Ses travaux portent sur la socio-économie du travail et de l'emploi, les relations sociales en entreprise et la justice sociale. II a récemment publié : "Animateurs vacataires et permanents: regards sur la qualité de l'emploi », Agora Débats/Jeunesses, $n^{\circ}$ 48, 2008 ; avec J.P. Yonnet, "Les attentes des salariés à bas salaires envers les syndicats: résultats d'une enquête par questionnaire ", La Revue de I'IRES, n 56, 2008 ; avec J.-M. Bonvin, Amartya Sen. Une politique de la liberté, Paris, Michalon, 2008. 
qualité de la formation mais aussi, d'un point de vue plus psychologique, sur la représentation du monde du travail (Forner et al., 1996). Leurs démarches de recherche de stage et leurs éventuelles difficultés à trouver une période de formation en entreprise ont jusqu'à présent été peu étudiées²

Notre étude visait à identifier les difficultés ressenties par les élèves dans leur recherche de stage, en partant de leur point de vue, pour le comparer ensuite avec celui des personnels enseignants et responsables de stage dans les établissements.

Deux hypothèses ont été posées. La première consistait à vérifier l'existence d'inégalités d'accès au stage pour les lycéens et étudiants professionnels, en essayant d'identifier les élèves concernés. La seconde hypothèse postulait que les acteurs scolaires ont des modes spécifiques d'intervention ou de suppléance pour aider dans leur recherche ces élèves les plus en difficulté. Comment les professeurs adaptent-ils leur aide en fonction des difficultés ressenties par les élèves ? Comment considèrent-ils justement ces difficultés exprimées?

Dans un premier temps, nous revenons ainsi sur la diversité des difficultés rencontrées par les lycéens professionnels dans l'accès au stage et nous posons la question de la présence de mécanismes discriminatoires, à partir du ressenti des élèves. La construction $\mathrm{du}$ questionnaire permet d'analyser la nature des difficultés ressenties; en effet, il distingue des facteurs habituels de difficulté d'accès au marché de l'emploi pour les jeunes d'autres éléments attribués (par les élèves eux-mêmes) à des caractéristiques personnelles potentiellement discriminantes.

Le croisement avec le point de vue des enseignants permet d'approfondir la problématique et de tester la seconde hypothèse. Dans quelle mesure un raisonnement en termes de difficultés ou de discrimination les

\footnotetext{
${ }^{2}$ Tous les enseignements professionnels comportent un stage ou une période de formation en entreprise obligatoires, conformément à la loi d'orientation sur l'éducation du 10 juillet 1989. L'accès au stage figure au même titre que toute procédure d'embauche dans le cadre de la loi de lutte contre les discriminations de 2001 (art. L. 122-45 Code du Travail). En ce qui concerne la situation des lycéens, voir le rapport de Dhume et al. (2006). Les travaux de Landrieux-Kartochian et Guillot-Soulez (2005) posent la question plus spécifique de la discrimination sexuelle dans le cadre des stages dans l'enseignement supérieur (universitaire).
}

conduit-il à modifier leur action ? En effet, la recherche du stage est un moment pédagogique de l'année scolaire: il s'agit d'un apprentissage des réalités du marché du travail, à côté des autres apprentissages fondamentaux et/ou techniques effectués en classe, directement liés au métier en tant que tel. L'élève professionnel « apprend» les rudiments de la confrontation avec le marché du travail. Cette logique pédagogique n'est pas sans poser problème. Si certaines difficultés sont propres à un processus d'apprentissage (ou d'essais et d'erreurs), et constituent donc un terrain d'action pour ces personnels, d'autres formes de difficultés sont le signe, pour les enseignants, de mécanismes discriminatoires sur lesquels ils n'ont pas prise.

Nous nous proposons, dans une deuxième partie, d'interroger ces difficultés d'accès au stage au regard de la logique pédagogique défendue par la plupart des équipes enseignantes ou responsables des stages dans les lycées. En quoi l'existence de discriminations à l'entrée en stage remet-elle en question ces préceptes pédagogiques? Quelle est la capacité des porteurs de ces derniers, c'est-à-dire les enseignants ou responsables de formation, à trouver une solution au problème de l'accès au stage ?

\section{LES DIFFICULTÉS À TROUVER UN STAGE}

Nous nous basons ici sur une recherche effectuée lors de l'année scolaire 2006-2007 sur les difficultés et les discriminations à l'entrée en stage pour les lycéens professionnels et étudiants de BTS (Farvaque et al., 2007). Cette enquête s'est appuyée sur un questionnaire adressé directement aux élèves et sur une série d'entretiens auprès des enseignants ou responsables des stages ${ }^{3}$.

La méthode s'appuie sur la question des difficultés à trouver un stage, pour cerner l'existence éventuelle de discriminations ressenties par certains élèves. L'enquête part donc du ressenti des élèves. La

\footnotetext{
${ }^{3} \mathrm{Le}$ rapport final inclut également des entretiens auprès d'employeurs des bassins d'emploi.
} 


\section{Encadré 1 \\ L'enquête}

L'analyse a d'abord porté sur le vécu des élèves, au moyen d'un questionnaire distribué à 2244 lycéens et étudiants dans 22 lycées de 5 départements, avec la répartition suivante : Ain (20\%), Loire-Atlantique (4\%), Marne (5\%), Nord (44\%) et Pas-de-Calais (27\%). Les lycées ont été contactés par courriel dans un premier temps. Une relance importante auprès des lycées professionnels de la région explique que les départements du Nord et du Pas-de-Calais soient majoritaires. Le département du Nord est représenté dans sa diversité, avec 12 lycées différents (grandes et petites villes, zones rurales, diversité géographique). L'échantillon inclut au total des lycées différents du point de vue de la réussite scolaire, en utilisant comme indicateur les taux bruts de réussite au baccalauréat et des taux attendus par établissement $\left({ }^{*}\right)$. La présence des départements de l'Ain, de la Loire-Atlantique et de la Marne a été motivée par l'existence préalable de zones-pilotes de mise en œuvre du projet Transfert (programme EQUAL financé par I'Union européenne) de sensibilisation à la question des discriminations. L'étude présentée ici constituait le premier volet (diagnostic) de ce programme.

Le questionnaire comportait trois grandes parties. Une première portait sur l'état civil, où était incluse originalité du questionnaire - une question concernant le lieu de naissance des parents. La seconde abordait la scolarité des élèves, en interrogeant notamment les motivations à l'orientation vers une filière professionnelle et des questions subjectives concernant les perspectives futures (sentiment que la formation offre des débouchés ou non, perspectives d'emploi futures jugées bonnes ou mauvaises), de manière à obtenir une première indication du jugement des élèves sur leur formation. La dernière partie portait sur l'expérience individuelle en matière de recherche de stages professionnels. Nous nous sommes intéressés à la recherche de stage la plus récente, qu'elle concerne le stage pour l'année scolaire en cours, le cas échéant, ou le dernier stage effectué. On demandait aux élèves si ce stage avait été facile ou difficile à obtenir, avant de proposer une série de raisons dans le cas où une difficulté était exprimée. Les méthodes de recherche, le nombre de candidatures et de refus, et enfin l'appréciation subjective du stage (quand il a déjà été réalisél, étaient interrogés. La construction générale du questionnaire visait à aborder le parcours de recherche de stage en partant des difficultés ressenties, pour éventuellement relever des motifs, attribués par les élèves eux-mêmes, d'ordre discriminatoire.

Par ailleurs, nous avons effectué 35 entretiens téléphoniques avec des enseignants ou chefs de travaux dans différents lycées de ces départements (n'ayant pas nécessairement participé au questionnaire). La grille d'entretien portait sur l'appréciation, par les enseignants, des caractéristiques sociologiques des élèves (quel milieu, quels parcours, pourquoi une orientation vers les filières professionnelles ?), avant d'aborder la place des stages dans leur formation et les méthodes de recherche utilisées par les élèves. Une place importante dans l'entretien était ainsi consacrée aux élèves qui ne trouvent pas de stage : quel est le rôle de l'établissement dans ce cas? Pourquoi ces difficultés? Y a-t-il des éléments discriminatoires? Quelles sont les relations entre l'école et les entreprises? etc. La seconde partie de cet article reviendra plus longuement sur le point de vue des acteurs scolaires, que l'on comparera avec celui des élèves tel qu'il ressort du questionnaire.

Composé d'un peu plus de garçons (54\%) que de filles (46\%), âgés en moyenne d'un peu plus de 18 ans, l'échantillon des lycéens professionnels se répartit en trois tiers entre les niveaux CAP (Certificat d'aptitude professionnelle) et BEP (Brevet d'études professionnelles), les $1 \mathrm{re} /$ Terminale Bac pro, et enfin les BTS $\left({ }^{* *}\right)$.

Les trois principales filières représentées sont comptabilité/secrétariat (21\%), commerce/vente (17\%) et industrie (17\% également), suivies des métiers de l'électro-technique, de l'hôtellerie-restauration ou de la santé et du social (entre 7 et $10 \%)(* * *)$. 


\section{(encadré 1 : suite)}

Le niveau d'études des parents de ces élèves est en grande majorité inférieur au baccalauréat. Environ trois parents sur quatre sont nés en France métropolitaine. Le quart restant de parents nés hors France métropolitaine vient principalement du Maghreb (12-13\%), d'un autre pays européen (3\%) ou d'un pays africain hors Maghreb. Nous utiliserons par la suite un indicateur synthétique distinguant les élèves dont les deux parents sont nés en France métropolitaine (70\% des élèves) et ceux dont au moins un parent est né hors France métropolitaine $(30 \%)(* * * *)$. L'origine est une cause de discrimination possible mais ce n'est pas la seule. Si une attention particulière est accordée à cette variable ici, il convient de ne pas négliger l'analyse d'autres formes de discriminations, liées à l'âge, au sexe, au handicap, à l'orientation sexuelle, etc.

$\left(^{*}\right)$ : En distinguant les résultats pour les filières «Services » et «Industrie », on constate ainsi qu'une moitié des établissements obtient des taux de réussite supérieurs au taux attendu par académie, et une autre moitié des taux inférieurs.

(**) : Les étudiants de BTS ont été incorporés à l'analyse car ils sont rattachés à des lycées (d'enseignement général ou professionnel), même s'ils font partie de l'enseignement supérieur. On parlera donc des «lycéens » de façon générale et par extension, en y incluant les étudiants de BTS. Ceci conduit donc à avoir un échantillon spécifique, intégrant des niveaux d'étude très différents, dont il faudra tenir compte au moment des interprétations. En termes de représentativité de l'échantillon, on notera que la répartition filles/ garçons est identique à la répartition nationale (54,6\% de garçons en 2005-2006 pour l'ensemble des établissements publics et privés, soit 724000 élèves ; 56,8\% si l'on ne prend en compte que les établissements publics, soit 569000 élèves). Chiffres du ministère de l'Éducation nationale. $C f$. «Les élèves du second degré », in Repères et références statistiques - 2006, disponible sur : http:// www.education.gouv.fr/pid316/reperes-et-references-statistiques.html.

$(* * *)$ : Par ailleurs, en ce qui concerne les filières professionnelles, la population est globalement représentative de la population nationale en enseignement professionnel. Selon les chiffres du ministère de l'Éducation nationale (Ibid.), on compte $43 \%$ d'élèves dans les spécialités de production et $57 \%$ dans les spécialités de services. Les proportions initiales de notre échantillon sont respectivement de $35 \%$ et $65 \%$. Nous avons redressé les données en fonction de la proportion nationale.

$(* * *)$ : Nous n'avons pas connaissance de données nationales à ce sujet permettant de juger la représentativité de notre échantillon.

méthodologie utilisée n'est pas de même nature que les enquêtes dites d'audit par couples ou «testing », basées sur l'envoi de séries de curriculum vitae incluant une modification, toutes autres choses étant égales par ailleurs, concernant un élément biographique pouvant être discriminatoire (nom, adresse, etc.). Étant donné que la recherche de stage repose très peu sur l'envoi de $\mathrm{CV}$, la méthode, pourtant plus objective, s'avérerait peu opérationnelle pour le présent objet d'étude.

La démarche subjective n'en reste pas moins informative car elle renseigne sur le vécu, par de jeunes élèves, des premiers pas dans le monde du travail. Comme l'indiquent de nombreux résultats qui seront commentés plus bas, ce vécu témoigne de difficultés ressenties importantes, attribuées dans certains cas par les élèves eux-mêmes à des caractéristiques personnelles de nature discriminatoire. En aucun cas le questionnaire ne peut donc prétendre apporter le caractère de la preuve d'éventuelles discriminations (la parole des enseignants venant cependant souvent corroborer leurs impressions de discriminations, ou relativiser certaines difficultés - nous y reviendrons dans la deuxième partie).

Nous présentons ci-dessous les principaux résultats du questionnaire, qui montrent des variations importantes du ressenti des difficultés entre les lycées et les filières. La nature même des difficultés varie selon les étudiants, avec une influence forte de l'origine des parents cumulée au milieu social. Or, c'est bien souvent le réseau social qui permet d'accéder facilement aux stages: pour les jeunes connaissant des difficultés d'accès au stage, l'école intervient en dernier recours.

\section{Des variations importantes selon le lycée et la filière}

Un jeune sur quatre de l'échantillon déclare avoir connu des difficultés pour obtenir un stage. La question était: "Ce stage a-t-il été facile ou difficile à obtenir? » Nous parlerons de taux de difficultés 
ressenties en rapportant le nombre d'élèves indiquant avoir eu des difficultés dans leur recherche de stage à l'ensemble des élèves. Les garçons sont plus nombreux que les filles à déclarer avoir ressenti des difficultés ( $28 \%$ contre $23 \%$ ). De même, la proportion de réponses positive à cette question augmente avec l'âge et le niveau de diplôme préparé.

Ainsi des variations frappantes apparaissent entre les 22 lycées où le questionnaire a été diffusé : des lycées affichent des taux de difficulté supérieurs à $40 \%$, d'autres aux alentours de $15 \%$ seulement, ce qui pourrait témoigner de stigmates portés par certains lycées, hypothèse qui nécessiterait d'autres matériaux empiriques pour être approfondie. Le taux de difficulté varie en fonction de la filière, de façon très significative (graphique 1). Les élèves des filières de spécialité production $(30 \%)$ sont plus nombreux à déclarer avoir connu des difficultés que les élèves des filières services $(23 \%)$. Plus précisément, c'est dans la filière électrotechnique que les jeunes disent être dans ce cas (1 sur 3), suivie des filières santé-social, industrie (30\%) et comptabilité/ secrétariat $(28 \%)^{4}$.
L'activité et le niveau d'études des parents sont significatifs (plus le niveau scolaire est bas, plus les difficultés sont grandes). Par exemple, $35 \%$ des élèves dont la mère n'a pas suivi d'études font état de difficultés. Si aucun des parents ne travaille, le taux de difficulté est de $30 \%$, contre $23 \%$ si les deux parents travaillent.

Le lieu de naissance des parents apparaît également très significatif : $22 \%$ des enfants dont les parents sont nés en France métropolitaine ont ressenti des difficultés, contre $33 \%$ des enfants dont un parent au moins est né hors France métropolitaine (tableau 1). Les enfants d'un parent né au Maghreb ou dans un autre pays africain sont presque deux fois plus nombreux que les enfants dont les parents sont nés en France métropolitaine à déclarer avoir rencontré des difficultés (39\% contre $22 \%$ ). Le fait d'être

\footnotetext{
${ }^{4}$ La filière « autre » regroupe des formations tertiaires : informatique, assurance, etc. La filière « artisanat» concerne ici principalement les métiers de bouche (boucherie, boulangerie, etc.) et les fleuristes.
}

Taux de difficultés ressenties par filière, en $\%$

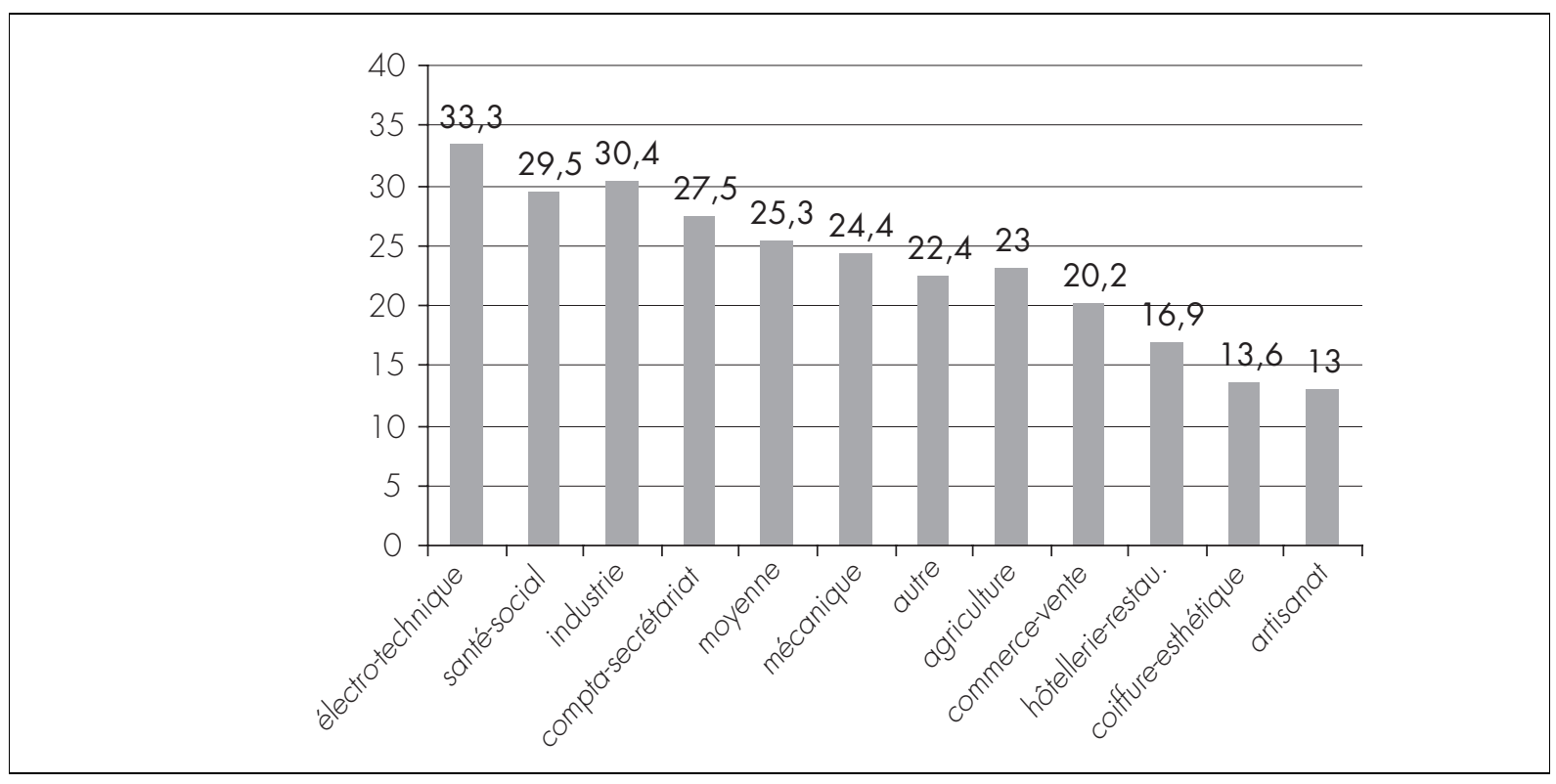

Taux de difficultés ressenties : nombres d'élèves ayant déclaré avoir eu des difficultés à trouver un stage/ensemble des élèves (Question : «Ce stage a-t-il été facile ou difficile à obtenir ? ». Modalités : Très facile, plutôt facile, plutôt difficile, très difficile).

Lecture : $33 \%$ des élèves de la filière électrotechnique disent avoir éprouvé des difficultés à trouver un stage.

Source : enquête ORSEU; champ : 244 lycéens de 22 lycées. 
Tableau 1

Difficultés ressenties en fonction de l'origine des parents

\begin{tabular}{|l|c|c|c|}
\hline \multicolumn{1}{|c|}{ Lieu de naissance des parents } & \multicolumn{2}{|c|}{ Pourcentage de jeunes déclarant avoir eu des } \\
difficultés
\end{tabular}

Lecture : $45 \%$ des élèves dont un des deux parents au moins est né dans un pays du Maghreb disent avoir eu des difficultés à trouver un stage. Source : enquête ORSEU.

Tableau 2

Régression logistique sur la difficulté ressentie pour obtenir un stage

\begin{tabular}{|c|c|c|c|}
\hline & \multirow[b]{2}{*}{ Modalité } & \\
\hline & & Probabilité & Effectifs \\
\hline Lieu naissance père & $\begin{array}{l}\text { France métropolitaine } \\
\text { Hors France métropolitaine }\end{array}$ & $\begin{aligned} & \text { Réf. } \\
+ & 29 \% *\end{aligned}$ & $\begin{array}{r}1491 \\
516\end{array}$ \\
\hline Lieu naissance mère & $\begin{array}{l}\text { France métropolitaine } \\
\text { Hors France métropolitaine }\end{array}$ & $\begin{array}{l}\text { Réf. } \\
\text { (n.s.) }\end{array}$ & $\begin{array}{r}1538 \\
469\end{array}$ \\
\hline Activité père & $\begin{array}{l}\text { Travail } \\
\text { Hors travail (inactif, chômeur ou retraité) }\end{array}$ & $\begin{array}{l}\text { Réf. } \\
\text { (n.s.) }\end{array}$ & $\begin{array}{r}1545 \\
462\end{array}$ \\
\hline Activité mère & $\begin{array}{l}\text { Travail } \\
\text { Hors travail (inactif, chômeur ou retraité) }\end{array}$ & $\begin{array}{l}\text { Réf. } \\
\text { (n.s.) }\end{array}$ & $\begin{array}{r}1095 \\
912 \\
\end{array}$ \\
\hline Langue & $\begin{array}{l}\text { Le français est ma langue maternelle } \\
\text { Le français n'est pas ma langue maternelle mais je } \\
\text { le maîtrise correctement } \\
\text { J'ai des difficultés à parler le français }\end{array}$ & $\begin{array}{l}\text { Réf. } \\
+37 \% \\
+108 \%\end{array}$ & $\begin{array}{r}1752 \\
238 \\
17\end{array}$ \\
\hline
\end{tabular}

Le seuil de significativité des probabilités est de $5 \%$, sauf* (10\%); (ns. : non significatif).

Lecture : par rapport à un élève dont le père est né en France métropolitaine, un élève dont le père est né hors France métropolitaine a, toutes choses égales par ailleurs, une probabilité supérieure de $29 \%$ d'avoir ressenti des difficultés à trouver un stage.

Source : enquête ORSEU.

une fille ou un garçon conduit également à des variations importantes dans ces réponses; ainsi, les garçons d'origine étrangère sont en proportion nombreux à avoir ressenti des difficultés. Ainsi, $45 \%$ des garçons d'origine maghrébine et $53 \%$ des garçons d'origine africaine hors Maghreb ont ressenti des difficultés.
L'influence de l'origine est double. Elle témoigne de difficultés plus grandes liées à l'apparence ou au nom ou prénom, c'est-à-dire des difficultés liées à des motifs discriminatoires. Elle renvoie aussi à la situation sociale des familles de notre échantillon originaires de l'étranger, en particulier de l'Afrique, où le niveau d'études des parents est plus bas (encore 
plus en ce qui concerne le niveau d'études maternel), où la part des parents qui ne travaillent pas augmente, ainsi que la taille des familles (nombre de frères et sœurs). Ces différents facteurs rendent la recherche plus difficile, essentiellement par manque de capital social, culturel et économique des parents.

Ainsi, l'influence de l'origine sur cet indicateur subjectif de difficulté est importante. En outre, plus encore que le lieu de naissance des parents (surtout le lieu de naissance du père), la maîtrise de la langue explique largement le fait de déclarer avoir ressenti ou non des difficultés (tableau 2). Les variables socio-économiques concernant les parents (activité professionnelle, niveau d'études, etc.) jouent un rôle plus significatif pour expliquer les méthodes de recherche que le ressenti des difficultés, comme on le verra plus loin.

Les types de difficultés ressenties varient en fonction des caractéristiques individuelles et de la famille, comme on le voit ci-dessous. L'exploration des résultats du questionnaire permet de poser l'hypothèse de la discrimination, à partir du ressenti des élèves.

\section{Des difficultés différentes selon le milieu social et l'origine des parents}

\section{$24 \%$ des élèves déclarent avoir rencontré des difficultés}

Environ 540 élèves sur l'ensemble des 2244 déclarent avoir connu des difficultés plus ou moins grandes dans la recherche du stage. Un peu moins (435) ont précisé les obstacles rencontrés (avec en moyenne 2,3 motifs donnés par individu).

Nous avons choisi de proposer, dans le questionnaire, des motifs renvoyant de façon générale aux difficultés probables en matière de recherche de stage pour un public jeune et avec peu d'expérience (timidité, ne pas connaître d'employeurs, absence de moyen de transport, etc.), en les mêlant à des motifs liés plus directement à des critères sélectifs basés sur l'âge, l'apparence (tenue vestimentaire, poids), mais aussi à des motifs possibles de discrimination (sexe, couleur de peau, nom et prénom comme critères discriminants sur une lettre de candidature, lieu de résidence comme stigmate, etc.).
Le graphique 2 présente les motifs de difficulté identifiés par les jeunes eux-mêmes (en ne retenant que les jeunes ayant déclaré avoir ressenti des difficultés), en distinguant selon le sexe. Les motifs retenus pour la population totale sont comparés avec ceux choisis par la sous-population des jeunes ayant une origine étrangère réelle ou supposée.

En ce qui concerne l'ensemble des jeunes ayant ressenti des difficultés dans leur recherche de stage (graphique à gauche), la première raison, invoquée dans plus d'un cas sur deux, est le fait de ne pas connaître d'employeur. Les deux obstacles qui suivent sont l'absence de moyen de transport et la timidité (qui sont plus fréquemment cités par les filles). La problématique des transports et des déplacements est en effet importante, mentionnée par de nombreux professeurs et chefs de travaux dans les entretiens (élèves trop jeunes ou pas assez fortunés pour avoir le permis de conduire, insuffisance des transports en commun en zone rurale). Les motifs discriminatoires apparaissent ensuite : le lieu de résidence est cité par un jeune sur cinq ayant connu des difficultés, puis la couleur de peau et le nom ou prénom. Dans l'ensemble, les trois premiers motifs donnés renvoient à des éléments assez classiques pour un public jeune et inexpérimenté, comme on l'a dit plus haut.

Les choses changent assez nettement quand on regarde le sous-échantillon des jeunes dont un parent au moins est né hors France métropolitaine.

\section{Avec un parent au moins né hors de France : des difficultés ressenties liées à la couleur de peau ou au nom}

Ces jeunes sont sur-représentés dans la part des jeunes ayant eu des difficultés. Comme on l'a montré ci-dessus, $33 \%$ d'entre eux disent avoir rencontré des difficultés, contre $67 \%$ qui estiment que le stage a été facile ou très facile à obtenir (contre $22 \%$ et $78 \%$ pour les jeunes dont les parents sont tous deux nés en France).

La partie droite du graphique 2 montre l'ordre d'importance des différents obstacles rencontrés dans la recherche du stage. Pour les jeunes dont un parent est né hors France métropolitaine et qui déclarent 
Graphique 2

Obstacles rencontrés dans la recherche du stage par les élèves de lycée professionnel (élèves ayant ressenti des difficultés)

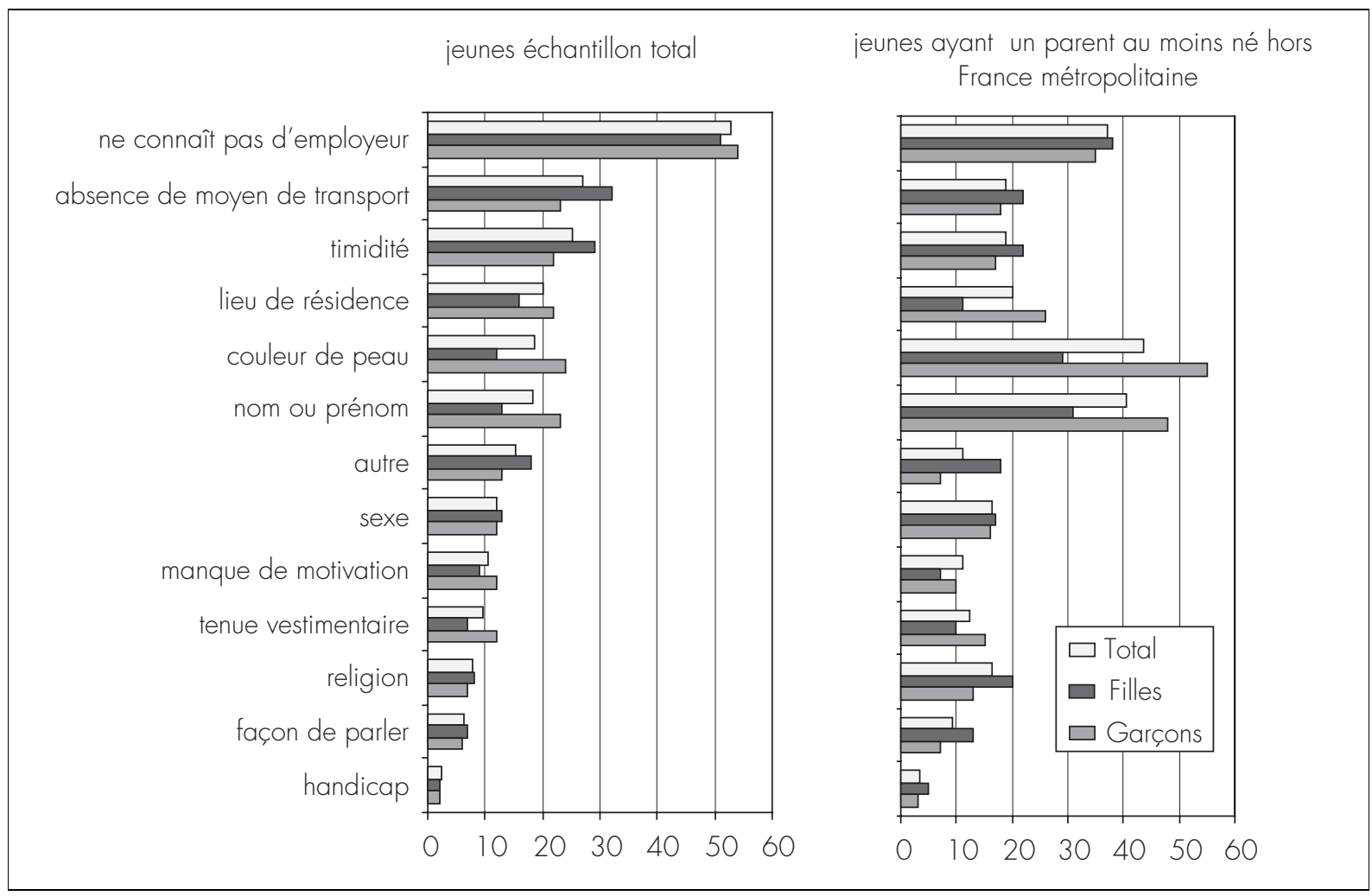

Pourcentages supérieurs à 100 , les répondants pouvant donner plusieurs motifs.

Note de lecture : Parmi les élèves ayant ressenti des difficultés pour accéder à un stage, $53 \%$ déclarent que c'est parce qu'ils ne connaissent pas d'employeur (respectivement $51 \%$ pour les filles et $54 \%$ pour les garçons) ; parmi les jeunes ayant un parent au moins né hors de France métropolitaine, ils ne sont plus que $37 \%$ à évoquer ce motif (respectivement $37 \%$ pour les filles et $35 \%$ pour les garçons).

Champ : élèves déclarant avoir ressenti des difficultés $(\mathrm{N}=539)$ et élèves dont un parent au moins est né hors France métropolitaine et déclarant avoir ressenti des difficultés $(\mathrm{N}=208)$.

avoir rencontré des difficultés à obtenir un stage, la couleur de peau et la consonance du nom ou prénom viennent en premier. Plus de $40 \%$ d'entre eux disent devoir leurs difficultés à l'un de ces motifs (et plus fréquemment en ce qui concerne les garçons). Le fait de ne pas connaître d'employeur vient compliquer la recherche. Les motifs qui apparaissaient plus haut dans la hiérarchie pour l'ensemble de l'échantillon (comme l'absence de moyen de transport ou la timidité) sont moins souvent cités : le problème ne réside plus à ce niveau, selon les lycéens et étudiants interrogés.

Par ailleurs, plus l'obstacle concerne un élément de nature discriminatoire, plus le degré de difficulté ressenti est grand (réponses "très difficile" plus fréquentes). Pour les autres obstacles, le degré de difficulté exprimé est moins fort (réponses «plutôt difficile »).

\section{Rechercher un stage : le réseau et l'école comme recours}

De l'étude du nombre de candidatures à un stage et du nombre de refus, il ressort que l'origine des élèves semble constituer un facteur de difficulté. Dans l'ensemble, un lycéen ou étudiant sur deux s'est contenté d'une ou deux demandes auprès d'entreprises pour trouver un stage. Une forte majorité des élèves ( $42 \%)$ n'a subi aucun refus, ce qui témoigne d'une recherche bien ciblée et efficace. Les jeunes dont un parent est né hors France métropolitaine doivent envoyer plus de candidatures aux entreprises, 
et ce de façon très significative. $52 \%$ des enfants dont les parents sont tous les deux nés en France métropolitaine ne font qu'une ou deux candidatures ; en comparaison, les enfants d'un parent au moins né hors France métropolitaine ne sont plus que $41 \%$ dans ce cas. Seuls $13 \%$ des premiers font plus de 10 candidatures; ils sont plus de $21 \%$ pour les seconds. De même, la probabilité de subir des refus augmente pour les jeunes dont un parent est né hors France métropolitaine. En particulier, ils sont près de $11 \%$ à avoir subi plus de dix refus, contre un peu plus de $6 \%$ pour les jeunes dont les deux parents sont nés en France métropolitaine.

Nos données confirment que les jeunes d'origine étrangère doivent contacter plus d'entreprises, pour des résultats pourtant moins souvent positifs ${ }^{5}$. Ceci est lié au plus faible réseau parental, et donc à l'obligation de se débrouiller soi-même, sans que l'on ne possède nécessairement les bons «codes» de la recherche. Ceci semble également témoigner de l'incorporation, dans le comportement de recherche de stage, de l'appartenance à une catégorie subissant régulièrement des discriminations dans l'accès aux entreprises, et en conséquence d'une exigence de devoir en faire plus.

L'activation du réseau familial (contacts personnels) facilite la recherche de stage, tandis que les recherches basées sur l'envoi de candidatures spontanées semblent plus ardues. Le fait que l'un des parents au moins ne travaille pas réduit les chances d'obtenir le stage par des contacts personnels (tableau 3). Les variables socio-économiques, essentiellement l'activité professionnelle des parents, apparaissent ici très significatives, alors que les variables concernant le lieu de naissance des parents ne le sont plus 6 .

\footnotetext{
${ }^{5}$ On retrouve un résultat mis en avant par Viprey et Deroche (2000). Les auteurs observaient que la durée d'obtention d'un contrat en alternance était beaucoup plus courte chez les jeunes Français ou Européens que chez les jeunes des autres catégories d'origine. $C f$. aussi Viprey (2002)

${ }^{6}$ Le rôle du réseau personnel dans l'accès au stage pour les étudiants du supérieur a été étudié par Landrieux-Kartochian et Guillot-Soulez (2006) sur un échantillon d'étudiants de Licence 3 et Mastère 1 de la Sorbonne. Les auteurs montrent que l'activation du réseau est le moyen de recherche le plus efficace si l'on considère un certain nombre d'indicateurs (rapidité d'obtention, conformité des stages avec le projet professionnel, etc.). Cette étude montre également que les jeunes femmes ont une capacité de sollicitation du réseau inférieure aux jeunes hommes. Notre régression va dans le même sens.
}

Dans le cas où les deux parents travaillent, $38 \%$ des élèves ont obtenu leur stage grâce à des contacts personnels; ce taux chute à $25 \%$ quand les deux parents ne travaillent pas. Il est en revanche plus probable que les jeunes dont les parents ne travaillent pas aient obtenu leur stage par l'entremise de l'école ou d'un professeur : ils sont $25 \%$ dans ce cas, contre moins de $15 \%$ seulement pour les élèves dont les deux parents travaillent. Il semble bien que l'école intervienne comme dernier recours pour les élèves ayant eu des difficultés.

Le fait d'obtenir son stage grâce à l'intervention de l'établissement scolaire témoigne ainsi de la difficulté à l'obtenir par des canaux plus directs, comme le démarchage, et peut également signaler l'absence d'un réseau personnel et familial. En proportion, les jeunes d'origine étrangère réelle ou supposée passent plus souvent par l'intermédiaire de l'école ; à l'inverse, les enfants dont les parents sont nés en France métropolitaine ont plus souvent recours aux contacts personnels. Le facteur véritablement significatif est ici moins l'origine que le milieu social des parents.

Ainsi, l'inactivité parentale a un effet significatif sur le fait de passer par l'école, toutes choses égales par ailleurs (probabilité supérieure de $25 \%$ ). Les professeurs sont sollicités à mesure que le nombre de refus augmente.

L'aide des professeurs semble donc bénéficier en priorité à ceux qui déclarent avoir des difficultés liées au manque de capital social familial et à l'origine des élèves. Quel est le point de vue des enseignants sur cette logique de suppléance? Comment perçoivent-ils les difficultés que ressentent les élèves, et de quelle façon interviennent-ils pour aider ces élèves?

\section{TROUVER UN STAGE : QUELS PRINCIPES D'INTERVENTION DES ACTEURS SCOLAIRES?}

Plus d'une trentaine d'entretiens téléphoniques ont été réalisés avec des personnels des établissements scolaires (chefs de travaux, enseignants, proviseurs, etc.). Souvent placés à l'interface entre les élèves et 
Tableau 3

Régression logistique sur l'obtention du stage via les contacts personnels

\begin{tabular}{|c|c|c|c|}
\hline & \multirow[b]{2}{*}{ Modalité } & \\
\hline & & Probabilité & Effectifs \\
\hline Sexe & $\begin{array}{l}\text { Masculin } \\
\text { Féminin }\end{array}$ & $\begin{array}{l}\text { Réf. } \\
-24 \%\end{array}$ & $\begin{array}{l}983 \\
858\end{array}$ \\
\hline Lieu naissance père & $\begin{array}{l}\text { France métropolitaine } \\
\text { Hors France métropolitaine }\end{array}$ & $\begin{array}{l}\text { Réf. } \\
\text { (n.s.) }\end{array}$ & $\begin{array}{r}1361 \\
480 \\
1408\end{array}$ \\
\hline Lieu naissance mère & $\begin{array}{l}\text { France métropolitaine } \\
\text { Hors France métropolitaine }\end{array}$ & $\begin{array}{l}\text { Réf. } \\
\text { (n.s.) }\end{array}$ & $\begin{array}{r}433 \\
1414 \\
\end{array}$ \\
\hline Activité père & \begin{tabular}{|l} 
Travail \\
Hors travail (inactif, chômeur ou retraité)
\end{tabular} & $\begin{array}{l}\text { Réf. } \\
-24 \% *\end{array}$ & $\begin{array}{r}427 \\
1000\end{array}$ \\
\hline Activité mère & $\begin{array}{l}\text { Travail } \\
\text { Hors travail (inactif, chômeur ou retraité) }\end{array}$ & $\begin{aligned} \text { Réf. } \\
-28 \% \\
\end{aligned}$ & $\begin{array}{r}841 \\
1610 \\
\end{array}$ \\
\hline Langue & $\begin{array}{l}\text { Français langue maternelle } \\
\text { Français pas langue maternelle, mais maîtrise } \\
\text { Difficultés à parler français }\end{array}$ & $\begin{array}{l}\text { Réf. } \\
\text { (n.s.) }\end{array}$ & $\begin{array}{r}1610 \\
231\end{array}$ \\
\hline Études actuelles & $\begin{array}{l}\text { BTS (Brevet de technicien supérieur) } \\
\text { BEP (Brevet d'études professionnelles)/CAP } \\
\text { (Certificat d'aptitude professionnelle) } \\
\text { BAC pro }\end{array}$ & $\begin{array}{l}\text { Réf. } \\
\text { (n.s.) } \\
-30 \%\end{array}$ & $\begin{array}{l}673 \\
593 \\
572\end{array}$ \\
\hline Filière & \begin{tabular}{|l|} 
Production \\
Services
\end{tabular} & $\begin{aligned} & \text { Réf. } \\
&- 27 \% \\
&\end{aligned}$ & $\begin{array}{r}613 \\
1228 \\
\end{array}$ \\
\hline $\begin{array}{l}\text { Nombre de demandes } \\
\text { de stage }\end{array}$ & $\begin{array}{l}\text { 1-2 demandes } \\
3-5 \text { demandes } \\
5-10 \text { demandes } \\
+ \text { de } 10 \text { demandes }\end{array}$ & $\begin{array}{l}\text { Réf. } \\
\text { (n.s.) } \\
-54 \% \\
-94 \%\end{array}$ & $\begin{array}{l}903 \\
367 \\
288 \\
283\end{array}$ \\
\hline Nombre de refus & $\begin{array}{l}\text { Aucun refus } \\
1-2 \text { refus } \\
3-5 \text { refus } \\
+ \text { de } 5 \text { refus }\end{array}$ & $\begin{array}{l}\text { Réf. } \\
-53 \% \\
\text { (n.s.) } \\
\text { (n.s.) }\end{array}$ & $\begin{array}{l}762 \\
412 \\
292 \\
375\end{array}$ \\
\hline
\end{tabular}

Le seuil de significativité des probabilités est de $5 \%$, sauf* $(10 \%)$; (n.s. : non significatif).

Lecture : par rapport à un garçon, une fille a, toutes choses égales par ailleurs, une probabilité inférieure de $24 \%$ d'avoir trouvé son stage grâce à des contacts personnels.

Source : enquête ORSEU.

les entreprises, les enseignants et surtout les chefs de travaux ${ }^{7}$ ont un rôle crucial. Quelles sont les stratégies employées par ces acteurs pour réduire les difficultés des élèves dans leur recherche et faire en sorte que chacun trouve un stage?

\footnotetext{
${ }^{7}$ Dans les lycées techniques et professionnels, le chef de travaux joue un double rôle d'organisateur et de conseiller du chef d'établissement, tant pour l'enseignement initial que pour la formation continue. Il est généralement en charge des relations avec les entreprises (pour trouver des lieux de stage, vérifier que l'apprentissage en entreprise se passe correctement, etc.). Il peut conserver une activité d'enseignement. C'est généralement vers eux que nous avons été orientés lors de l'enquête.
}

Deux principales logiques d'action émergent des entretiens menés: une logique pédagogique et une logique de placement. La logique pédagogique vise à inculquer aux jeunes la démarche de recherche d'emploi via ces premières initiations et confrontations aux exigences du monde de l'entreprise. Au cours de cette logique individualisée, l'ampleur et la nature des difficultés ressenties et exprimées par les élèves sont ainsi évaluées; de cette évaluation découlent les modalités d'intervention de l'établissement. La logique de placement, ensuite, vient en complément. Elle vise à ne laisser aucun élève sans 
stage. Il s'agit donc d'une intervention en dernier ressort. Ces deux logiques, qui s'articulent parfois difficilement, sont successivement abordées dans les deux prochaines sous-parties.

Elles s'inscrivent dans une séquence égalitariste. Celle-ci se fonde d'abord sur un principe d'égalité des moyens, sous-tendant la logique pédagogique et entretenu par une démarche de responsabilisation " positive" des élèves, sauf dans certains cas rares ${ }^{8}$. Le second temps de la séquence se fonde sur une action supplémentaire d'égalisation des résultats, en donnant priorité aux élèves les plus en difficulté ou discriminés. Les intermédiaires agissent alors dans une logique de placement, dont l'objectif est d'éviter qu'un élève ne se retrouve sans stage.

\section{Une logique pédagogique}

La logique pédagogique se base donc sur une logique d'égalité de moyens (fournir les mêmes moyens à tous) et une démarche de conseils, d'activation de la recherche, d'individuation. On peut parler de responsabilisation pédagogique, à l'image de ce que l'on observe dans les instances d'insertion des jeunes en difficultés comme les missions locales (Farvaque, 2005) : la confrontation avec le marché du travail est un processus d'apprentissage ; l'institution reste en soutien et s'appuie sur les leçons de cette confrontation pour apprendre au jeune à améliorer ses démarches. Le moment du stage, dans les lycées professionnels, institue autour du jeune une démarche d'insertion professionnelle qui emprunte selon nous à cette logique de responsabilisation; celle-ci comporte toutefois une grande part de pédagogie, basée sur l'idée qu'il n'y a d'apprentissage qu'actif, en situation. L'immaturité de certains élèves, leur naïveté, ou encore leur méconnaissance de la réalité du monde du travail peuvent être combattues par cette confrontation pédagogique, l'ambition du personnel scolaire étant alors de leur inculquer certains codes (présentation de soi, notamment vestimentaire), ce qui s'apparente à un appren-

\footnotetext{
${ }^{8}$ On parlera de responsabilisation " positive » au sens d'action de l'institution sur les personnes en vue d'accroître leur capacité d'action, leur autonomie (c'est en ce sens que la démarche est pédagogique), par opposition à une démarche de responsabilisation «négative » qui correspondrait à une vision punitive, qui n'a pas été rencontrée dans l'étude.
}

tissage par l'expérience. À un niveau individualisé, l'évaluation de la motivation et du caractère actif de la recherche est centrale.

La croyance en l'individu reste forte ; elle peut toutefois s'avérer complètement inadéquate en cas de discriminations, en particulier fondées sur l'origine des jeunes. Parfois, comme le dit un conseiller pédagogique interrogé, le cas de ces jeunes qui ne trouvent pas de stage alors qu'ils n'ont «aucune raison manifeste» de ne pas en trouver témoigne de discriminations :

«Il ne faut pas nier la réalité qui est là : on a plein de Noirs, d'Arabes, d'Asiatiques, qui ne trouvent pas de stage, quelle que soit leur motivation. On trouve toujours pour eux, on arrive toujours à les caser, il n'y en a pas un qui reste sur le carreau, mais le problème est réel. C'est sûr, il y a ceux qui ne cherchent pas sérieusement, qui s'habillent n'importe comment, et pour eux ce n'est pas la couleur de peau qui compte. Le vrai problème est qu'il y a plein d'élèves qui n'ont pas de raison manifeste de ne pas trouver de stage, et qui se retrouvent sans rien. Ce sont des élèves qui ont cherché sérieusement, mais qui n'ont rien trouvé. Si j'en ai 20 qui sont dans ce cas, il y en a 16, 18, qui sont d'origine étrangère ou de couleur... " (Conseiller principal d'éducation, Loire-Atlantique).

Ici, les difficultés de recherche ne sauraient être attribuées au comportement de recherche de l'élève ou à sa responsabilité individuelle. Parler de "difficultés » euphémise considérablement la situation et masque les structures de contraintes 9 . Encore que, convaincus du bien-fondé de l'approche pédagogique, et voulant ainsi maintenir un territoire professionnel d'intervention légitime, certains responsables enseignants soulignent qu'il n'est pas toujours évident de démêler la part de responsabilité individuelle de celle attachée à des facteurs extérieurs à la personne, sur lesquels on ne peut agir. En d'autres termes, pour conserver une intervention possible sur l'élève l'intervention à un niveau plus global (inégalités sociales, représentations des employeurs, comportements racistes, etc.) étant exclue, car soit impossible,

\footnotetext{
${ }^{9}$ Cette notion (Folbre, 1994) renvoie au biais d'inégalité systématique ou permanente que subit un groupe dans la société. $C f$. aussi Tinker (1990).
} 
soit trop lourde, perdue d'avance, ou hors du corps de métier de ces acteurs -, certains responsables cherchent à ne pas dédouaner totalement les élèves d'une certaine responsabilité dans leur recherche. Un chef de travaux d'un lycée de la métropole lilloise insiste bien sur les difficultés plus grandes pour les jeunes d'origine étrangère et pense qu'on peut en effet parler dans certains cas de discriminations: "ça doit arriver malgré tout, il n'y a pas de raison qu'on échappe à la règle...» Toutefois, selon lui, «on fait face à des attitudes pas très claires de certaines entreprises, mais on a aussi des jeunes qui ne sont pas toujours très coopératifs ». Une chef de travaux d'un autre lycée nordiste va dans le même sens: "Oui, c'est vrai que certains ont des difficultés, mais d'autres n'en ont pas du tout. Donc on ne peut pas dire. Les entreprises, elles ne nous donnent pas le motif quand elles refusent une demande, donc on ne peut pas savoir si c'est pour cela ou autre chose. Parfois, on peut se demander. Mais on ne peut pas dire que les difficultés sont spécifiquement liées au nom ou prénom, ou à la couleur de peau. Le problème, c'est surtout un problème de manque d'énergie pour trouver un stage. " Le fait que certains jeunes ne subissent pas de difficultés particulières pour obtenir un stage prouve au moins, à écouter ces responsables des stages, que la discrimination n'est pas totale, mais qu'il n'est pas possible de nier le problème. Une interprétation de ces propos pourrait être qu'avec de la bonne volonté (de "l'énergie»), on s'en sort toujours. Cette vision «responsabilisante» repose sur une grande confiance en l'individu ${ }^{10}$. Le fait que la discrimination ne fonctionne pas à cent pour cent crée l'espace nécessaire pour que surgissent des croyances en la capacité des jeunes à vaincre le phénomène.

\section{Une logique de placement}

Que font les enseignants et responsables d'éducation? Comme on l'a dit plus haut, après la logique selon laquelle les jeunes sont guidés dans leur recherche (obligation de moyens), survient une logique de placement, où la responsabilité des établissements

${ }^{10}$ Une fiction nécessaire, en quelque sorte, comme le dit Dubet (2004). devient prégnante, et la (pseudo) responsabilité des élèves est délaissée. Cette seconde logique est indépassable : elle renvoie à l'obligation qui est faite aux personnels enseignants de trouver un stage (et donc de se comporter comme "placeurs ») et non plus seulement de développer une pédagogie de la première confrontation avec le monde du travail ${ }^{11}$.

La logique de placement est celle de l'« obligation». Ce terme est revenu à de nombreuses reprises et possède un double sens : à la fois une obligation légale et bureaucratique (les intervenants évoquent le «Bulletin officiel», «les textes», etc.), mais également, semble-t-il, une obligation morale, qui dicte l'action. "On n'a pas le choix», dit une chef de travaux tertiaires du Nord. "Le stage est obligatoire dans le cursus, donc on est obligé de leur trouver un terrain de stage $»$. Notons que cette obligation est le plus souvent évoquée pour les lycéens, moins pour les étudiants après le bac (les étudiants en BTS demeurant dans le périmètre d'action du lycée). La division des responsabilités est claire pour un chef de travaux : "Pour les BEP et les Bac pro, le stage est de la responsabilité de l'équipe pédagogique: au bout du compte, nous devons fournir le terrain de stage. Pour les BTS, la recherche est plus de la responsabilité des étudiants, c'est une démarche d'étudiant, plus de lycéen. On se doit quand même de fournir les moyens, mais il n'y a plus la même obligation de résultats ».

Cette obligation de trouver un stage implique donc, pour les acteurs scolaires, d'intervenir auprès des élèves qui n'en ont pas trouvé, pour une raison ou une autre, dans le sens d'une obligation de résultats. Mais comme le disent certains, ils se mobiliseront plus pour l'élève discriminé que pour l'élève qui n'a pas fait d'efforts. «Pour (...) ceux qui se sont décarcassés mais qui n'ont rien trouvé, c'est pour eux

\footnotetext{
${ }^{11}$ Un lycée situé dans un quartier sensible a refusé de diffuser le questionnaire au motif que les élèves ne connaissent pas de difficultés, car ils n'ont pas à chercher de stage par eux-mêmes (la logique " pédagogique » étant ici inexistante). Une formation professionnelle est garantie avec un stage « clés en main », comme le dit le proviseur. La logique de placement est proposée sans détour, afin d'éviter les effets négatifs d'une recherche autonome qui n'aboutirait pas. "On n'a jamais laissé un gosse tout seul, on connaît très bien les humiliations au début, au moment de la recherche de stage», explique le proviseur: "Le but de l'école, c'est de leur donner une formation, de leur montrer qu'ils sont capables. On leur évite le passage par l'humiliation».
} 
qu'on va travailler en premier, pas pour les autres...» (chef de travaux tertiaires et enseignante en secrétariat, Nord). Ce mode d'intervention n'intervient de toute façon, se justifient certains chefs de travaux interrogés, qu' « en dernier recours ».

Le premier principe de responsabilisation pédagogique continue de produire ses effets, car nombreux sont les personnels scolaires qui mettent en avant la capacité des élèves à trouver par eux-mêmes un stage, quand bien même l'expérience montre qu'ils cumulent des facteurs généralement sources de difficultés plus grandes dans leurs relations avec les entreprises. Cette analyse se base sur les discours des acteurs, ce qui ne signifie pas que les actes reproduisent parfaitement ces discours. En d'autres termes, on peut penser que l'insistance récurrente sur cette première phase pédagogique, que nous qualifions également de responsabilisante et d'éducative, remplit une fonction de légitimation d'un territoire professionnel de la part de ces acteurs (profs, chefs de travaux, etc.), quand on les interroge sur le sujet des discriminations. Pour eux, dire qu'il n'y a rien à faire face aux inégalités d'accès au stage, voire face aux comportements discriminants, serait inconcevable, voire un aveu d'échec.

\section{Quelle obligation de résultats?}

L'obligation de résultats supplante au final l'obligation de moyens, laquelle risquerait de montrer ses limites devant la sélectivité à l'embauche et les difficultés évoquées jusqu'ici. L'approche égalitaire et républicaine de l'école conduit les enseignants à agir selon le principe d'une justice non pas méritocratique mais bien de résultats. Ils doivent s'assurer que personne ne reste « sur le carreau».

La logique d'égalité des résultats varie également selon ce que l'on entend par obligation de résultats : s'agit-il que chacun ait un stage, quel qu'il soit? Ou bien que chacun puisse obtenir un stage qui corresponde à son projet professionnel et qui lui offre un environnement d'apprentissage propice ? La seconde alternative, qualitative, est idéalement celle qui est préférée, mais en pratique, il est difficile de ne pas se replier sur une logique quantitative, surtout quand il y a urgence (par rapport au calendrier de l'année scolaire).
Pour les acteurs scolaires, placer les jeunes en entreprise ou leur trouver un stage ne va pas de soi. Cette quasi-obligation qui leur est faite exige la constitution d'un réseau d'entreprises, sur la base de garanties et de "contrats de confiance», pour ainsi dire, offerts par les établissements. Enseignants et chefs de travaux se doivent, outre leurs activités scolaires, d'établir des liens avec le tissu économique local (c'est l'une des tâches centrales du chef de travaux). Il s'agit notamment d'anticiper le moment où il faudra suppléer les élèves dans leur recherche de stage; dès lors, le maintien d'un réseau où les relations sont bonnes est primordial (Dhume, SagnardHaddaoui, 2006).

Tous les lycées fonctionnent avec un réseau d'entreprises qui constituent autant de lieux de stage. Ce réseau est activé au cas par cas, selon les problèmes rencontrés par les jeunes et le diagnostic posé par l'enseignant. L'activation de ce réseau répond parfois à une logique d'excellence (par exemple, des restaurants gastronomiques huppés vers lesquels pourront être orientés certains élèves talentueux), mais le plus souvent à une logique quantitative (trouver un stage à tous les élèves). L'action s'inscrit ici dans un souci d'égalité sur les résultats, parfois dans l'urgence (quelques jours avant la date de début des stages). Il s'agit cependant d'une solution provisoire à un problème temporaire (l'accès au stage d'une promotion particulière de lycéens) qui ne permet pas de combattre les mécanismes permanents de discriminations et d'inégalités d'accès au stage. Il est même possible qu'elle les renforce indirectement. Si la conservation du réseau est primordiale en effet, alors les intermédiaires peuvent indirectement reproduire de la sélectivité dans leurs « choix » d'orienter tel ou tel jeune vers telle ou telle entreprise du réseau. Une des craintes exprimées à plusieurs reprises par les chefs de travaux interrogés est le risque qu'un élève «flingue» le stage (en se comportant mal avec l'employeur, en abandonnant le stage, etc.), ce qui remet en cause toute la stratégie des chefs de travaux. «J'ai un réseau d'entreprises sous le coude, mais je ne veux pas leur mettre n'importe qui entre les mains ; je veux garder ma crédibilité ! » avoue un chef des travaux de l'Ain. Un réseau réduit d'une ou plusieurs entreprises anciennement partenaires signifie évidemment, dans un contexte marqué par la 
rareté des relations durables avec le tissu économique local, plus de difficultés pour les placeurs.

La place des intermédiaires (ici, les enseignants ou chefs de travaux) et leur possible participation au " système » de la discrimination sont ainsi interrogées. La prise en compte, par anticipation, des préférences des entreprises, peut conduire les responsables des stages dans les établissements à renforcer de façon «systémique " les discriminations. En effet, les représentants des établissements scolaires ne sont pas en position de force pour négocier avec les entreprises : " il existe une acceptation tacite de la discrimination du fait de la position de faiblesse des intermédiaires. Le contexte de pénurie d'emplois favorise la position de force des entreprises sur le marché du travail. Il est difficile pour un intermédiaire de résister aux exigences des employeurs car il risque de priver d'un emploi un autre jeune. Les organismes de formation en alternance sont confrontés aux mêmes difficultés » (Viprey, Deroche, 2000). Un chef de travaux déclare ainsi : "Quand une entreprise me dit qu'elle ne veut que des gens blancs, je souris gentiment, j'accepte. J'ai pas le choix, je dois trouver des lieux de stage, je ne vais pas me priver d'une entreprise qui accepte des stagiaires... (...) [Enquêteur : que peut-on faire?] Nous au lycée on ne peut rien faire... Mais quand un magasin me dit qu'ils ne veulent pas qu'on leur envoie des jeunes de couleur, je laisse tomber, je préfère placer une petite blanche... On a environ $15 \%$ de gens de couleur dans l'établissement, surtout d'origine maghrébine, mais ça veut dire que les autres il faut bien aussi leur trouver des stages... ». Le rapport de force entre intermédiaires publics et entreprises conduit à une acceptation de la discrimination, voire à une co-production.

L'approche quantitative des stages, consistant à éviter qu'un élève ne « reste sur le carreau », doit être interrogée du point de vue de la qualité (professionnelle, éducative, etc.) du stage lui-même. L'obligation juridique, exprimée dans les textes officiels, ne se limite pas à l'obtention d'un stage quel qu'il soit : tout stage doit correspondre à un référentiel précis. C'est ce que rappelle ce chef de travaux du Pas-deCalais, tout en confirmant le dilemme qui peut étreindre certains enseignants : «Le stage doit être validé par le prof de toute façon; dans la convention du BO [Bulletin officiel], le stage doit correspondre au référentiel. Mais c'est vrai que quand le stage a tendance à ne pas arriver, on va avoir tendance à accepter plus ou moins tout et $n$ 'importe quoi... Il est évident qu'il y a des stages plus ou moins intéressants. »

Par rapport à un stage obtenu par relations personnelles, un stage obtenu, en dernier ressort, par l'établissement, a toutes les chances d'être de moindre qualité. L'évaluation de cette qualité peut se baser sur des éléments objectifs, comme le niveau de rémunération, mais il s'agit d'un indicateur plus pertinent pour les stages du supérieur que pour les stages lycéens. Des éléments subjectifs inclus dans notre questionnaire permettent de pointer des niveaux de satisfaction différents en ce qui concerne des aspects précis du stage, selon la méthode d'obtention. Ces aspects concernent par exemple l'adéquation du contenu du stage avec la formation («ce stage m'a permis de mettre en pratique ma formation »), le sentiment d'avoir été «bien suivi» ou encore «bien intégré à l'entreprise». Il est possible de montrer (régression logistique non reprise ici) que le fait d'avoir ressenti des difficultés d'accès au stage et d'être passé par l'intermédiaire de l'école pour finalement obtenir ce stage conduit à un jugement négatif sur la période en entreprise. Par exemple, toutes choses égales par ailleurs, être passé par l'école augmente de $39 \%$ la probabilité de s'être senti mal intégré dans l'entreprise. Les résultats du modèle de régression logistique montrent ainsi la significativité des difficultés ressenties et du passage par l'établissement (mais également de l'origine) pour expliquer le sentiment de ne pas avoir été bien intégré dans l'entreprise. De valeur illustrative, il appelle des compléments empiriques pour analyser plus précisément la qualité des stages dans le secondaire et le supérieur post-baccalauréat.

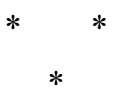

L'article a souligné les difficultés propres à ces premières confrontations avec le marché du travail que représentent les stages pour les lycéens professionnels, et les modalités de réponse des enseignants. Bien que subjectives, les informations recueillies 
concernant les difficultés d'accès au stage donnent à voir, avec une grande régularité, une concentration de difficultés chez les élèves de certains milieux sociaux. L'accès au stage diffère selon l'origine sociale. Les facteurs liés à l'origine et au milieu social se cumulent pour expliquer le sentiment de difficultés et le recours à l'aide de l'établissement (par opposition aux élèves pouvant activer le réseau familial).

Les logiques d'action des professionnels scolaires ont été évoquées en interrogeant leur sens; elles permettent de trouver un stage pour tout le monde à défaut de trouver des stages de qualité pour chacun. La séquence égalitariste retenue (égalité de moyens mâtinée de responsabilisation pédagogique, puis égalité des résultats en termes d'obtention d'un stage, quel qu'il soit ou presque) mérite d'être débattue.

À tout le moins, elle doit s'inscrire dans une réflexion plus générale sur l'enseignement professionnel (et sa dévalorisation), les relations écoleentreprises et cet objet difficilement identifiable que sont les stages pour les lycéens professionnels. Une des questions centrales et non réglées tient à l'isolement des enseignants ou chefs de travaux : livrés à eux-mêmes devant des demandes de tri préalable ou de présélection des stagiaires de la part de certains employeurs, sans outils et mal équipés dans cette négociation, ils sont fréquemment conduits, tant bien que mal, à reproduire des mécanismes « systémiques » de discrimination. Leur territoire légitime d'action se limite ainsi souvent à la pédagogie de la motivation et de la responsabilisation, quand les difficultés d'accès au stage découlent de facteurs plus sociaux.

Il reste à dépasser cette approche du ressenti qui peut poser parfois le problème de la délimitation entre représentations individuelles et contraintes effectives. Ces données subjectives restent toutefois un matériau important et intéressant pour l'analyse, d'autant plus que c'est en partie à partir de ce matériau - ce que disent les élèves - que travaillent effectivement les acteurs scolaires. En partie seulement toutefois, car ce qui leur est dit est systématiquement croisé avec d'autres variables; comme le déclare un responsable scolaire, nombre d'étudiants n'ont pas de « raison manifeste » de ne pas trouver de stage, ce qui implique donc d'aller au-delà des facteurs individuels (motivation, etc.) pour interroger les facteurs sociaux, tant en termes d'influence du milieu social familial que de la prévalence de contraintes supplémentaires et de mécanismes discriminatoires dans l'accès à l'entreprise. À ce titre, l'analyse des relations entre école et entreprises mérite d'autres approfondissements. L'alternance entre deux mondes régis par des règles aussi distinctes ne se décrète pas; elle se construit progressivement, à un niveau local, dans une logique précaire. Les logiques de construction des besoins des entreprises en matière de stages professionnels, d'un côté, les logiques de construction des besoins des établissements scolaires en matière d'insertion en stage, de l'autre, et surtout la coordination de ces besoins, peuvent constituer autant de pistes de recherche.

\section{Bibliographie}

Dhume F., Sagnard-Haddaoui N. (2006), La discrimination, de l'école à l'entreprise. La question de l'accès aux stages des élèves de lycée professionnel en région Lorraine, ISCRA, programme TALENT (FSE, PIC EQUAL).

Domingo P. (2002), «Logiques d'usage des stages sous statut scolaire», Formation Emploi, $\mathrm{n}^{\circ} 79$, juillet-septembre, pp. 67-81.
Dubet F. (2004), L'école des chances: qu'est-ce qu'une école juste?, Paris, Seuil, «La République des idées ».

Farvaque N. (2005), «L'expérience de la Bourse d'Accès à l'emploi pour les jeunes en difficulté : responsabilité individuelle, responsabilité collective et liberté de choix », in C. Baron, E. Dugué et 
P. Nivolle (éds.), La place des jeunes dans la cité. De l'école à l'emploi ?, Paris, L'Harmattan, pp. 191-207.

Farvaque N., Broumm E., Messaoudi D. (2007), Les discriminations à l'entrée en stage : l'expérience des lycéens et étudiants en filière professionnelle, le point de vue des enseignants et maîtres de stage, ORSEU, Rapport pour la Fédération Léo Lagrange et l'UNSA, février.

Folbre N. (1994), Who pays for the kids? Gender and the Structure of Constraint, New York: Routledge.

Forner Y., Dosnon O., Gonnin-Bolo, Lebeaume J. (1996), «Les stages en entreprise : quels effets chez les jeunes de collège ? », Revue Française de Pédagogie, $\mathrm{n}^{\circ} 115$, pp. 33-42.

Landrieux-Kartochian S., Guillot-Soulez C. (2005), «Les stages, une des formes de la discrimination sexuelle dans le monde du travail? », Cahiers $d u$ CERGORS, université Paris-1, $\mathrm{n}^{\circ} 3$, septembre.

Landrieux-Kartochian S., Guillot-Soulez C. (2006), Réseaux et insertion dans le monde du travail : le cas des stages, XVII ${ }^{e}$ Congrès de l'AGRH, Reims Management School, novembre.

Tinker I. (1990), Persistent Inequalities, New York : Oxford University Press.

Viprey M., Deroche L. (2000), « Conditions d'accès à l'entreprise des jeunes étrangers ou d'origine étrangère : nature des résistances ", Migrations Études, mai-juin-juillet, ${ }^{\circ} 94$.

Viprey M. (2002), « Les mécanismes de discrimination à l'égard des jeunes dont l'origine étrangère est réelle ou supposée », La Revue de l'IRES, n³9, pp. 61-84.

\section{Résumé}

\section{Discriminations dans l'accès au stage : du ressenti des élèves à l'intervention des enseignants \\ Nicolas Farvaque}

L'accès au stage en entreprise pour les lycéens et étudiants professionnels en France est ici étudié à partir d'une enquête auprès des élèves et des enseignants. Dans un premier temps, l'analyse se concentre sur les types de difficultés rencontrées par les élèves dans leur recherche et aux discriminations éventuelles que certains d'entre eux pensent subir. Ces difficultés ressenties sont ensuite interrogées au regard des logiques d'intervention des équipes enseignantes ou responsables des stages dans les lycées: une logique pédagogique, soutenant les efforts de chaque élève dans sa recherche de stage, et une logique de placement, visant à suppléer ceux pour qui cette recherche n'a pas abouti.

Mots clés

Lycée professionnel, inégalités, stage de formation, données statistiques

Journal of Economic Literature : I 29, J 71 